\title{
La incorporación de la perspectiva de género en la docencia universitaria del Grado en Relaciones Laborales y Recursos Humanos de la Universitat de València ${ }^{1}$
}

\section{Empar Aguado Bloise ${ }^{a}$, Elena García Testal ${ }^{b}$}

${ }^{a}$ Departamento Sociología y Antropología Social, Universitat de València; Correo electrónico: Teresa.Empar.Aguado@uv.es, bepartamento de Derecho del Trabajo y de la Seguridad Social, Universitat de València; Correo electrónico:elena.garcia@uv.es

\section{Abstract}

We present in this paper the interuniversity Teaching Innovation Project (TIP) "Incorporar la Perspectiva de Gènere en la Docència Universitària del Grau en Relacions Laborals com a element d'Innovació $i$ Pràctica de bona ciència» developed at the University of Valencia.

This TIP was born with the intention of giving impetus to the implantation of the gender perspective, in a gender mainstreaming way, in the teaching of the Degree in Labor Relations and Human Resources. Incorporating the Gender Perspective is necessary to train professionals in the world of work. Comprehensive gender analysis is a cutting-edge element of innovation that is managing to provide necessary and more democratic responses to our societies.

The activity designed to incorporate this perspective into teaching has the involvement of a team who teach in this Degree for a long period of time, know the Degree perfectly and are involved in its improvement. The design of the TIP will allow the adoption of direct measures to incorporate gender in both ordinary and complementary teaching, obtaining professionals in the labor sciences with a better and broader training by being able to incorporate the necessary gender perspective.

Keywords: teaching, gender, labor relations, human resources, equality.

\section{Resumen}

En esta Comunicación presentamos el Proyecto de Innovación Docente (PID) interuniversitario "Incorporar la Perspectiva de Gènere en la Docència Universitària del Grau en Relacions Laborals com a element d'Innovació i Pràctica de bona ciència» desarrollado en la Universitat de València.

Este PID nace con la intención de dar impulso a la implantación de la perspectiva de género, de forma transversal, en la enseñanza del Grado en Relaciones Laborales y Recursos Humanos. Incorporar la Perspectiva de Género es necesario para formar profesionales en el mundo del trabajo. Los análisis de género con carácter integral son un elemento de Innovación de última generación que están logrando dar respuestas necesarias y más democráticas a nuestras sociedades.

La actividad diseñada para incorporar esta perspectiva a la docencia cuenta con la implicación de profesores y profesoras que imparten docencia en este Grado durante un largo periodo de tiempo,

\footnotetext{
${ }^{1}$ Esta investigación forma parte del proyecto "Incorporar la Perspectiva de Gènere en la Docència Universitària del Grau en Relacions Laborals com a element d'innovació i pràctica de bona ciencia (INNOVAGEN)", financiado en el marco de la convocatoria de Proyectos de Innovación Educativa para el curso 2019-2020 UV-SFPIE_PID19-1097490 de la Universitat de València.
} 
conocen perfectamente el Grado y están implicadas en su mejora. El diseño del PID permitirá adoptar medidas directas de incorporación del género tanto en la docencia ordinaria como la complementaría, consiguiendo profesionales de las ciencias del trabajo con una mejor y más amplia formación al poder incorporar la necesaria visión de género.

Palabras clave: docencia, género, relaciones laborales, recursos humanos, igualdad. 


\section{Introducción: la necesidad de incorporar la perspectiva de género en la docencia: carencias, innovación y práctica}

La perspectiva de género en la docencia universitaria hace referencia a una política de promoción de la igualdad de género y la diversidad aplicada como estrategia transversal, de tal forma que sean tenidos en cuenta intereses, necesidades y características de mujeres y hombres, partiendo de la necesidad de señalar la existencia de roles y estereotipos construidos culturalmente y atribuidos al género.

Como sostiene la historiadora de la ciencia Londa Schiebinguer (2008), creadora del proyecto Gendered Innovations $^{2}$, los análisis de género en las diferentes disciplinas y con carácter transversal está abriendo nuevas vías para la innovación y la excelencia. Y, más concretamente plantea aprovechar el poder creativo del análisis de género en la ciencia. Este proyecto liderado por esta investigadora ha alcanzado dimensión de colaboración internacional y logrado el apoyo de instituciones como la Comisión Europea, que en 2011 creó un grupo de expertos para unirse al proyecto (Magallón, 2016: 57).

La transversalidad de la perspectiva de género ha sido recogida a nivel normativo como un principio de actuación que obliga a los poderes públicos a tener en cuenta las necesidades e intereses tanto de las mujeres como de los hombres en todas las estructuras, procesos y políticas. Pese al prolífico marco normativo sobre perspectiva de género en la docencia universitaria, su implementación todavía no ha sido efectiva (Verge y Alonso, 2019)

Una docencia ciega al género no puede ser el ejemplo de una docencia universitaria, pues creemos en la necesidad que tiene la docencia en la universidad de ofrecer al alumnado conocimientos y herramientas no solo para comprender sino también para intervenir en el mundo a través de su futuro ejercicio profesional y por ende en el conjunto de la sociedad. Sin embargo, la realidad es que continúa desarrollándose una docencia ajena a esta necesidad. Así se ha puesto de referencia por diferentes estudios que se han aproximado a la cuestión (Verge y Cabruja, 2017). La investigación realizada por estas autoras puso de manifiesto que la incorporación efectiva de la perspectiva de género aún era un reto pendiente, así como que las universidades -y, en consecuencia la docencia-, no escapan de la reproducción de roles y de estereotipos, en la segregación de las responsabilidades, en la infrarrepresentación de las mujeres en las categorías más altas de la carrera académica, en la dificultad de reconocer el liderazgo femenino, en la invisibilización de las mujeres y sus aportaciones (Verge y Cabruja, 2017).

La autopercepción de que, en nuestro entorno universitario, y en concreto en el Grado en Relaciones Laborales y Recursos Humanos, sucedía aún hoy algo similar nos impulsó a liderar una iniciativa que persigue la generación de redes y el agrupamiento por parte de docentes de diferentes áreas de conocimiento implicadas en el desarrollo de este grado en la Universitat de València. Esta asociación está orientada a la consecución y el avance de un objetivo común tanto de diagnosticar la situación y percepción real de partida en el Grado, como de constituir una herramienta para compartir las experiencias individuales, con el objetivo final de incorporar de forma efectiva la perspectiva de género en la docencia de este Grado.

La Global University Network for Innovation (GUNI, 2017), de la cual forma parte la UNESCO, presentó en marzo de 2017 su informe Higher Education in the world: Towards a Socially Responsible University. Dicho informe recoge los Objetivos de Desarrollo Sostenible (ODS), un conjunto de 17 prioridades que sirven como hoja de ruta para las universidades socialmente responsables. El objetivo 5 centra su atención en la igualdad de género (Agencia por la Calidad del Sistema Universitario de Cataluña, 2018).

\footnotetext{
${ }^{2}$ Para mayor información sobre este proyecto puede acceder al siguiente enlace: $<$ https://genderedinnovations.stanford.edu/ $>$
} 
El Proyecto de Innovación Docente (PID) que presentamos se sitúa en consonancia con los objetivos del III Plan de Igualdad de la Universitat de València, cuyo eje 2 se refiere a la voluntad de la Universitat de València de transversalizar la perspectiva de género en la enseñanza y garantizar la investigación con perspectiva de género. En este sentido, se plantea la difusión de las aportaciones de las mujeres en la investigación y el fomento de la docencia con perspectiva de género junto con la elaboración de materiales docentes de referencia.

Para llevar a cabo esta innovadora propuesta de práctica docente que pone el foco en la formación en perspectiva de género, concurrimos a la convocatoria de ayudas del Vicerrectorado de Empleo y Programas Formativos, de la Universitat de València, para el desarrollo de Proyectos de Innovación para el curso 20192020 (convocada por Resolución de 10 de junio de 2019). En este Programa de la Universitat de València -Proyectos de Innovación Docente (PID)- se buscaba el desarrollo de Proyectos de innovación de carácter general, encaminados a la implementación de metodologías docentes, instrumentos metodológicos y técnicas de enseñanza-aprendizaje, de carácter innovador.

El PID presentado fue seleccionado y obtuvo una ayuda económica del mencionado Vicerrectorado de Empleo y Programas Formativos de la Universitat de València para el desarrollo de algunas de las actuaciones programadas, que analizaremos en los apartados siguientes.

\section{Objetivos}

El objetivo principal del Proyecto de Innovación Docente INNOVAGEN es proporcionar al alumnado las herramientas que les permitan la identificación de los estereotipos y roles de género, lo que contribuirá al desarrollo de su espíritu crítico y la adquisición de las competencias necesarias para que su práctica profesional futura se desarrolle con conciencia y perspectiva de género; todo ello con la finalidad de tratar de formar profesionales en el mundo del trabajo que compartan una visión de la igualdad real, que consideramos que en los actuales planes de estudio se produce de forma incompleta, como señalaremos a continuación.

Podemos sistematizar nuestros objetivos en siete grandes líneas:

OBJETIVO 1: Generar materiales y recursos educativos para que el profesorado pueda avanzar en la implementación sistemática de la perspectiva de género en la docencia universitaria del Grado en Relaciones Laborales y Recursos Humanos.

OBJETIVO 2: Generar una red estable de profesorado sensible a la incorporación en la perspectiva de género en la docencia universitaria del Grado en Relaciones Laborales y Recursos Humanos.

OBJETIVO 3: Generar interacciones y retroalimentación a través de la transferencia de experiencias consolidadas en la interacción y aplicación de este proceso de innovación llevado a cabo en las aulas del Grado en Relaciones Laborales y Recursos Humanos.

OBJETIVO 4: Generar sensibilidad en el estudiantado para combatir los estereotipos de género inherentes en nuestra cultura y presentes en los entornos educativos y laborales para poder combatirlos.

OBJETIVO 5: Avanzar en la generación de una red interuniversitaria con el objeto de poder intercambiar experiencias con el profesorado de distintas universitarias y disciplinas que estén emprendiendo experiencias similares. 
OBJETIVO 6: Servir de elemento de reflexión sobre la posición que ocupan las mujeres en los entornos profesionales del Grado en Relaciones Laborales y Recursos Humanos.

OBJETIVO 7: Diagnosticar la ceguera de género y la percepción sobre la misma por parte del profesorado y del alumnado implicado en la acción.

Pero para situar el objetivo de nuestro Proyecto de Innovación Docente consideramos necesario explicar las cuestiones más relevantes del Grado en Relaciones Laborales y Recursos Humanos en la Universitat de València, para trasladar la complejidad de estos estudios, así como la importancia de incorporar la perspectiva de género de forma efectiva.

\subsection{El Grado en Relaciones Laborales y Recursos Humanos en la Universitat de València: el estudio}

\section{del trabajo humano y la perspectiva multidisciplinar}

El Grado en Relaciones Laborales y Recursos Humanos de la Universitat de València tiene por objetivo la adquisición de la formación necesaria para comprender de una forma integrada la complejidad y el carácter dinámico e interrelacional del trabajo humano, atendiendo las perspectivas jurídica, organizativa, psicológica, sociológica, histórica y económica. Además, promueve el espíritu crítico ante problemas sociales y laborales, la responsabilidad y el sentido ético para la aplicación y la defensa de los derechos fundamentales, la igualdad de oportunidades, la no discriminación y los valores democráticos. De acuerdo con la descripción que de estos estudios se hace en la web de la propia Universitat de València ${ }^{3}$ parece imprescindible que el acercamiento al fenómeno del trabajo humano en el siglo XXI se realice desde una perspectiva de género.

Sin embargo, una aproximación a la Memoria verificada del Grado en Relaciones Laborales y Recursos Humanos de la Universitat de València ${ }^{4}$ nos devuelve un resultado desolador, pues permite comprobar las escasas ocasiones en que se hace referencia al término "género", y a la igualdad entre hombres y mujeres.

Así, entre las Competencias Generales (CG en adelante) sólo encontramos una referencia en relación a esta cuestión. Se trata concretamente de una competencia general y una específica: la CG9 - Capacidad para respetar y promocionar los derechos fundamentales, la igualdad entre hombres y mujeres, la igualdad de oportunidades y la no discriminación, los valores democráticos y la sostenibilidad; y, la Competencia específica, la CE14 - Analizar y evaluar los factores que determinan las desigualdades en el mundo del trabajo -no específicamente vinculada a la igualdad por razón de género-, y algunas referencias más o menos concretas en los resultados de aprendizaje y en los contenidos, que listamos a continuación:

1. Como resultado de aprendizaje:

○ Asignatura: Economía Aplicada: - Conocer y analizar las cuestiones relativas a la igualdad de género y no discriminación entre hombres y mujeres en el ámbito económico.

- Asignatura: Teoría de las relaciones laborales: - Conocer las aportaciones teóricas existentes sobre las interdependencias entre la división sexual del trabajo y la construcción social de las identidades de género; y - Conocer los principales factores y dimensiones que determinan las relaciones sociales de producción y las desigualdades en el mundo del trabajo.

\footnotetext{
${ }^{3}$ Se puede consultar este enlace para mayor información $<$ https://www.uv.es/uvweb/universidad/es/estudios-grado/ofertagrados/oferta-grados/grado-relaciones-laborales-recursos-humanos-1285846094474/Titulacio.html?id=1285847461424>[Consulta: 15 de marzo de 2019]

${ }^{4}$ Se puede consultar este enlace para mayor información $<$ https://www.uv.es/graus/verifica/Relacions_Laborals/Memoria.pdf $>$ [Consulta: 15 de marzo de 2019]
} 
- Asignatura: Técnicas cualitativas de investigación social: - Ser capaz de identificar las formas de desigualdad sociolaboral y de segregación laboral de género, mediante indicadores sociolaborales de discriminación.

- Asignatura: Sistemas de Relaciones Laborales: -Analizar los factores de desigualdad sociolaboral y de discriminación laboral de género en relación con los contenidos y propuestas de medidas adoptadas en los procesos de negociación y concertación.

- Asignatura: Políticas sociolaborales, de empleo y de igualdad: - Conocer las políticas de igualdad en el ámbito sociolaboral, especialmente en lo referente a la igualdad de género y no discriminación entre hombres y mujeres. Y - Prestar especial atención a las políticas de igualdad y, en concreto, a los instrumentos normativos derivados de la Ley Orgánica para la igualdad efectiva de mujeres y hombres.

- Asignatura: La función de Recursos Humanos: -Ser capaz de diseñar, elaborar y evaluar planes de igualdad en las empresas.

- Asignatura: Prácticas Externas: - Ser capaz de aplicar en el ámbito laboral los derechos fundamentales y de igualdad entre hombres y mujeres.

2. Como contenido:

- Asignatura: Historia social y de las relaciones laborales contemporáneas: Los cambios sociales y laborales en el periodo de la II Revolución industrial y el capitalismo monopolista. El sufragismo y las políticas del trabajo de género

- Asignatura: Políticas sociolaborales, de empleo y de igualdad: Prestar especial atención a las políticas de igualdad y, en concreto, a los instrumentos normativos derivados de la Ley Orgánica para la igualdad efectiva de mujeres y hombres.

- Asignatura: Empleo y relaciones laborales en el mercado de trabajo: - Desigualdades sociales en el acceso al empleo. Empleo y género.

- Asignatura: Responsabilidad social corporativa en el ámbito de los recursos humanos: Políticas aplicadas de responsabilidad social corporativa: comités éticos, políticas de conciliación familiar/profesional, atención a discapacitados, igualdad de género, políticas de inserción, políticas de compras y subcontratación socialmente responsable, marketing y comunicación responsable.

De un total de 50 asignaturas que componen el Plan de Estudios del Grado, según la Memoria Verificada, sólo hemos identificado dos competencias (una CG y una $\mathrm{CE}$ ), y únicamente 10 asignaturas referían alguno de sus resultados de aprendizaje o de sus contenidos al género y la igualdad de oportunidades entre mujeres y hombres. No hemos identificado ninguna asignatura dedicada al diagnóstico, al análisis de las previsiones normativas o la relevancia de la igualdad por razón de género en el mundo del trabajo. A pesar de que la ley emite un mandato en el Conocimiento y la Educación Superior desde la perspectiva de género (Pérez Sedeño, 2018).

Este decepcionante panorama visible nos debe hacer cuestionar críticamente el Plan de Estudios Verificado, y nos conduce a plantearnos una batería de cuestiones: ¿es realmente ésta la realidad de la incorporación de la perspectiva de género en el Grado en Relaciones Laborales y Recursos Humanos en la Universitat de València? ¿Cuál es el desarrollo de la perspectiva de género incorporado a las Guías Docentes? ¿Es posible una revisión de las Guías Docentes desde la perspectiva de género para visibilizar los contenidos que efectivamente estamos impartiendo al alumnado? ¿Es posible incorporar la perspectiva de género únicamente desde las actividades complementarias desarrolladas como enseñanza adicional? 
En definitiva, con este contexto parece necesario realizar un esfuerzo para la innovación docente y la incorporación efectiva de la perspectiva de género en la formación de estos futuros profesionales, teniendo en cuenta que, según los organismos internacionales, la igualdad entre mujeres y hombres aún tardará un siglo en conseguirse.

Así, si centramos la atención en las asignaturas de contenido jurídico, se presenta como necesaria la incorporación de la perspectiva de género y variados los puntos de vista que pueden abordarse en la docencia de estas asignaturas. En primer lugar, el derecho del trabajo se ha ocupado solamente de un tipo de prestaciones de servicios a cambio de una contraprestación económica, ignorando otras fórmulas de trabajos o prestación de servicios casualmente realizados mayoritariamente por mujeres. En segundo lugar, hace falta incrementar la atención a las dificultades para lograr la igualdad derivadas de la doble o triple carga de las trabajadoras, y adoptar esta perspectiva en la explicación docente de las instituciones laborales o de seguridad social. En tercer lugar, debe incorporarse el análisis jurisprudencial, tanto para resaltar aquellas resoluciones que sí toman en consideración la perspectiva de género, como aquellas que manifiestan una interpretación no igualitaria. En cuarto lugar, explicar las instituciones de Seguridad Social desde la perspectiva de género, incluyendo el análisis crítico de la consideración de la maternidad como enfermedad, y la valoración como discriminatoria por razón de sexo que ello supone (Torres Diaz, 2018). En quinto y último lugar, es necesario explicar las normativas vigentes y las instituciones jurídicas desde la perspectiva de género, añadiendo como valor necesario los efectos negativos que sobre las mujeres puedan tener así como la necesidad de incorporar acciones positivas.

Por todo ello creímos imprescindible realizar dos tipos de actuaciones:

- analizar la incorporación efectiva de la perspectiva de género en las diferentes asignaturas que componen la docencia ordinaria del grado, a través de diferentes herramientas;

- y, simultáneamente, complementar la docencia en el aula con actividades complementarias que en unas ocasiones refuercen la perspectiva de género, y en otras subsanen la ceguera de género latente en las diferentes disciplinas del Grado.

\subsection{EI Proyecto de Innovación Docente (PID) INNOVAGEN de la Universitat de València}

El proyecto de innovación docente -INNOVAGEN- desarrollado en el curso 2019-2020 y respecto del que hemos solicitado renovación del PID para el curso 2020-2021- se caracteriza por su transversalidad: centra la atención en la perspectiva de género aplicada a la docencia universitaria, pero sobre todo es transversal por la manera en que lo hace: implicando a un equipo de profesorado mixto y paritario, presente en buena parte de las asignaturas del Grado, en todos los niveles académicos, que pertenece a diferentes departamentos, de diferentes facultades y universidades (interdepartamental, interfacultativo e interuniversitario). Los cuatro departamentos implicados, así como la composición del equipo del proyecto son estratégicos en el Grado puesto que desarrollan tareas de coordinación. La implicación de personas adscritas a cuatro departamentos diferentes (Derecho del Trabajo y de la Seguridad Social, Economía Aplicada, Dirección de Empresas y Sociología y Antropología Social) que están adscritos a tres facultades de la Universitat de València (Facultad de Ciencias Sociales; Facultad de Derecho y Facultad de Economía) hace que el carácter transversal gane fuerza y tenga mayor significado.

Las asignaturas del Grado en Relaciones Laborales y Recursos Humanos que en el curso 2019-2020 estuvieron implicadas en este Proyecto de Innovación Docente son las siguientes:

PRIMER CURSO: 33550 Derecho sindical; 33551 Economía aplicada; 33556 Sociología del trabajo; 33562 Derecho del trabajo I; 33558 Instrumentos y técnicas de información, organización del estudio y documentación; 33552 Introducción al funcionamiento de la empresa; 
SEGUNDO CURSO: 33553 La dirección en la empresa; 33557 Teoría de las relaciones laborales; 33563 Derecho del trabajo II; 33575 Economía del trabajo; 33568 Estructura de la organización y diseño de puestos de trabajo; 33566 Intervención administrativa en las relaciones laborales; 33560 Técnicas cualitativas de investigación social; 33559 Técnicas cuantitativas de investigación social;

TERCERO CURSO: 33564 Derecho de la seguridad social I; 33565 Derecho de la seguridad social II; 33570 Dirección estratégica de la empresa; 33574 Marco normativo y gestión de la prevención de riesgos laborales; 33576 Sistemas de relaciones laborales;

CUARTO CURSO: 33579 Prácticas externas; 33580Trabajo Fin de Grado; 33588 Dirección de la calidad y recursos humanos; 33597 Empleo público y relaciones especiales de Trabajo; 33582 Empleo y relaciones laborales en el mercado de trabajo valenciano; 33581 Sistemas laborales territoriales y desarrollo local; 33590 Responsabilidad social corporativa en el ámbito de los recursos humanos.

Además, el planteamiento basado en la innovación individual pero coordinada en cada asignatura y, posteriormente, la transferencia y el objetivo de compartir las buenas experiencias en una jornada de reflexión entre el profesorado y alumnado del Grado, permite establecer redes de trabajo y de retroalimentación continua en la consecución del objetivo principal: incorporar e implantar la perspectiva de género de forma transversal y efectiva en el Grado, creando una nueva cultura que se anticipe a la ceguera de género y a la producción y reproducción del conocimiento con bases androcéntricas y patriarcales.

Por otro lado, la incorporación de formación complementaria vinculada al género y el trabajo profundizará la formación del estudiantado de Grado, y lo preparará para el acceso al mundo del trabajo, espacio en el que las diferencias por razón de género tienen un carácter muy relevante.

La incorporación de expertas de otras universidades, que ya han implementado proyectos de innovación que incorporan la perspectiva de género en los estudios de Grado, facilitará una transferencia e intercambio de conocimiento muy provechoso.

En definitiva, el conjunto de actividades diseñadas, algunas ya desarrolladas y otras en proceso de desarrollo, permite crear una red de profesorado con formación y experiencia para, en un futuro próximo, proceder a la completa revisión de las Guías Docentes para la incorporación definitiva de la perspectiva de género en las enseñanzas del Grado en Relaciones Laborales y Recursos Humanos de la Universitat de València.

\section{Desarrollo de la innovación}

Para el desarrollo de la innovación docente hemos seguido las recomendaciones de las Guías elaboradas por la Xarxa Vives -Guies per a una Docència Universitària amb perspectiva de gènere- que se refieren a las siguientes acciones:

a. Diagnóstico de la conciencia de género y la percepción sobre la misma por parte del alumnado, así como del PDI implicado en la acción. Este objetivo se realizará mediante un cuestionario.

b. Incorporación del uso del lenguaje inclusivo y sensible al género, como instrumento de comunicación, así como creador de un imaginario simbólico y referencial del entorno convivencial.

c. Reflexiones críticas en relación al lenguaje jurídico.

d. Selección de ejemplos prácticos o materiales en la docencia para trasladar al alumnado, de una manera más conectada a la realidad, los conocimientos teóricos explicados en

2020, Universitat Politècnica de València 
clase: huyendo de ejemplos estereotipados que reproducen y refuerzan la socialización diferenciada del sistema sexo/género.

e. Manifestación sobre la importancia de los análisis críticos y visibilización numérica de los efectos en los sujetos de la elaboración de leyes o resolución de casos ciegos al género.

f. Análisis de casos - por vía interpretativa o aplicativa- desde la perspectiva de género.

g. Selección y creación de materiales: junto con los materiales típicos (leyes, sentencias, dictámenes, informes, etc.) que permiten trasladar al aula ejemplos relacionados con los contenidos teóricos explicados y que ayudan a tomar conciencia de los estereotipos y los sesgos de género.

De acuerdo con ello, hemos llevado a cabo el siguiente PLAN DE TRABAJO, que incluye una serie de acciones específicas, AGRUPADAS EN DOS BLOQUES:

\section{BLOQUE UNO: Transferencia de conocimiento «compartiendo experiencias y buenas prácticas»}

El profesorado incorpora a lo largo del periodo lectivo la perspectiva de género en la asignatura vinculada al PID, a través de las diferentes metodologías y estrategias. Finalizado el primer periodo de clases, se realizó una primera toma de contacto e intercambio de experiencias -24 de febrero de 2019- a través de un seminario interno entre el profesorado del PID, con la finalidad de organizar el resto de acciones vinculadas al PID, y especialmente, la realización de una Jornada de intercambio de experiencias fijada para el mes de noviembre de 2020, en el Salón de Actos de la Facultad de Ciencias Sociales. En esta jornada de intercambio contaremos con profesorado otras universidades con experiencias similares: Inmaculada Pastor (Directora del Observatori de la Igualtat de la Universitat Rovira i Virgili (URV), Anna Pérez Quintana (Directora de la Unitat d'Igualtat de la Universitat Rovira i Virgili (URV), M. José Díaz Santiago. Directora de la Unidad de Igualdad de Género de la Universidad Complutense de Madrid (UCM), y M. del Carmen Merino. Profesora de la Universitat de la Universidad de Valladolid (UVA). Debido a la situación sanitaria derivada del estado de alarma declarado el 14 de marzo de 2020 en relación a la COVID-19 esta jornada se trasladó del 28 de abril a noviembre de 2020, en que está prevista su realización on-line a través de la plataforma BBC.

Además, se ha elaborado un cuestionario con el objeto de diagnósticar la conciencia de género y la percepción sobre la misma, tanto del alumnado como del profesorado implicado en la acción. El cuestionario se implementará en el primer cuatrimestre del curso 2020-2021.

\section{BLOQUE DOS: Complementando la docencia ordinaria}

En este caso se han programado y realizado una serie de ACTIVIDADES COMPLEMENTARIAS CON PERSPECTIVA DE GÉNERO. Para llevarlas a cabo hemos contado con la colaboración presupuestaria de la Comisión Académica del Título y de la Facultat de Ciències Socials. Se han realizado las siguientes acciones:

1. Seminario sobre Necesidades de cuidados, trabajo de cuidados y políticas locales de atención a la dependencia. Personas Ponentes: Óscar Muñoz González (Programa de Doctorado en Ciencias Sociales de la Universitat de València) y Tatiana Sapena Escrivá (Confederación Sindical Comisiones Obreras del País Valencià). Profesorado Responsable: Empar Aguado Bloise (Prof. Sociología y Antropología Social, Universitat de València) y Josep Vicent Pitxer Campos (Prof. Economía Aplicada, Universitat de València), destinada a estudiantado de cuarto curso.

2. Seminario sobre El papel de las personas con perfil técnico en las políticas socioeconómicas locales que puso el foco en dos contenidos: el primero, Iniciativas y proyectos para la igualdad de género desde el sindicato, a cargo de Cristina Ochando Pardo (Técnica de igualdad, Secretaria de la Dona, Comisiones Obreras del País Valencià); y, el segundo, Las políticas socioeconómicas 
locales desde València Activa, a cargo de Juan Aparicio Guadas (Técnico de empleo, Fundació València Activa). Profesorado Responsable: Empar Aguado Bloise (Prof. Sociología y Antropología Social, Universitat de València) y Josep Vicent Pitxer Campos (Prof. Economía Aplicada, Universitat de València), destinada a estudiantado de cuarto curso.

3. Seminario: Mujeres y equipos directivos de pequeñas y medianas empresas de la Comunidad Valenciana, Profesora Responsable: Teresa Canet; Ponente Conferenciante: Da Concha Roig Lluesma.

4. Jornada sobre el acceso al empleo e identificación de puntos conflictivos desde una perspectiva de género: dignificación e igualdad, Profesores Responsables: Dra. Elena García Testal (Prof. Derecho del Trabajo, UV) Dr. Eduardo E. Taléns Visconti (Prof. Derecho del Trabajo, UV); Ponentes: Dra. Mercedes López Balaguer (Prof. Derecho del Trabajo, UV). “¿Es posible introducir medidas de acción positiva en el acceso al empleo"? Dra. María Gema Quintero Lima (Prof. Derecho del Trabajo, Universidad Carlos III de Madrid) "El empleo doméstico: dignificación del trabajo a través de la modificación normativa"; Dra. Elena García Testal (Prof. Derecho del Trabajo, UV) "Trabajadoras víctimas de violencia de género: ¿es posible mejorar sus opciones de empleo?"; Dra. Patricia Nieto Rojas (Prof. Derecho del Trabajo, Universidad Carlos III de Madrid) "Empleo y género en la negociación colectiva: ¿cómo puede intervenir positivamente en el empleo de las mujeres?";

5. Seminario sobre conciliación, corresponsabilidad y cuidados: Taller sobre la gestión de los derechos de conciliación en la empresa, Profesora Responsable: Elena García Testal (Prof. Derecho del Trabajo y de la Seguridad Social, Universitat de València). Ponente: Rafael Andrés Alcayde (Abogado especialista en Derecho Laboral; profesor asociado de la Universitat de València), destinada a estudiantado de segundo curso;

6. Seminario sobre género y sistema de seguridad social: destinada a estudiantes de $3 .^{\circ}$, con la incorporación de una especialista en la materia, Angustias Benito Benítez de la Universidad de Cádiz -sin fecha confirmada por razones de fuerza mayor y salud pública-.

7. Seminario sobre género y prevención de riesgos (como diseñar políticas de prevención de riesgos en el trabajo con perspectiva de género): La prevención de riesgos desde la perspectiva de género, Profesor Responsable: Cayetano Núñez Olmedo, Ponente María Teresa Miró (Universidad de Sevilla), destinada a estudiantado de tercer curso.

\section{Resultados}

Las fechas de entrega de esta comunicación -marzo de 2020- nos impide hacer una valoración más profunda de los resultados obtenidos en el curso 2019-2020.

La programación de actividades relevantes aún por desarrollar en este momento -docencia sin finalizar en el segundo cuatrimestre, actividades complementarias programadas, y un cuestionario de diagnóstico en proceso de elaboración -con la finalidad de evaluar la percepción del estudiantado sobre las competencias de género-, impiden hacer, en este momento, una valoración de los resultados obtenidos.

Pero sí cabe hacer una valoración previa. La solicitud del Proyecto de Innovación Docente, la movilización e implicación de tanto profesorado con docencia en este Grado, de diferentes departamentos, facultades y materias, han dado ya como frutos inmediatos la constitución de un grupo de reflexión sobre cuestiones pendientes de realizar, aspectos no abordados y propuestas de mejora en relación con la docencia realizada con perspectiva de género, que en sí mismas ya son positivas como punto de inicio de una verdadera 
innovación e incorporación de la perspectiva real del mundo de las relaciones laborales y del trabajo en la actualidad, sin invisibilizar una parte esencial de este panorama laboral, y sobre las consecuencias que las actuaciones realizadas redundan en la mejora de la calidad.

\section{Conclusiones y perspectivas de futuro}

Las conclusiones de las actuaciones realizadas hasta este momento, así como las perspectivas de futuro que hemos ido recogiendo desde la constitución del grupo de profesorado de este Proyecto de Innovación Docente son de varios tipos. La conclusión principal es la necesidad de una actuación urgente e intensa en el Grado en Relaciones Laborales y Recursos Humanos para poder formar profesionales completos y con una visión transversal del género en todos los aspectos de su actividad profesional. Las carencias de las actuaciones voluntariamente realizadas por el profesorado requieren de una unificación de actuaciones para su mejor implementación. La reflexión sobre contenidos, métodos, intereses y experiencias, interacciones y práctica docente, se constituyen en una herramienta necesaria e innovadora (Verge y Cabruja, 2017).

Hemos de señalar que en esta iniciativa hemos recibido el constante apoyo de la Comisión Académica del Título -apoyando formalmente el proyecto y subvencionando las actividades propuestas desde el PID- como de la Vicedecana de Igualdad de la Facultat de Ciències Socials, como de la Vicerrectora de Igualdad y Sostenibilidad de la Universitat de València.

El segundo bloque de conclusiones, más ambicioso, hace referencia a acciones de futuro, que al hilo de las actuaciones realizadas parece necesario afrontar en los próximos cursos académicos:

- Revisión de las Guías Docentes de las asignaturas del Grado en Relaciones Laborales y Recursos Humanos de la Universitat de València, con varios objetivos, que se señalan a continuación:

- visibilizar los aspectos relacionados con el género, así como la necesidad de incorporar más aspectos vinculados a la igualdad entre mujeres y hombres en cada una de las materias.

○ incrementar el número de autoras en las bibliografías -o si ya estuvieran, identificarlas con el nombre completo-.

○ diseño de mecanismos de evaluación de la adquisición de competencias en relación con el género.

- visibilizar la actividad profesional de las mujeres en cada una de las materias.

- Realización de una Guía para la incorporación de la perspectiva de género en los títulos de Grado en Relaciones Laborales y Recursos Humanos.

- Publicación de materiales en relación con las experiencias docentes y las propuestas de incorporación de la perspectiva de género en la docencia en el Grado en relaciones Laborales y Recursos Humanos.

- Transferencia de los conocimientos a los niveles de postgrado y en concreto al Máster en Dirección y Gestión de Recursos Humanos y al Máster en Prevención de Riesgos Laborales, de la Universitat de València, en el que los profesores que forman parte de este PID también imparten docencia.

- Revisión del Plan de estudios para incorporar -en realidad recuperar- asignaturas de contenido específico (y no sólo conformarse con el tratamiento transversal en el resto de asignaturas).

- Organización de formación específica al profesorado sobre cuestiones de género y sobre su incorporación a la docencia, como fórmula de expansión al resto de profesorado implicado en la docencia en el Grado en Relaciones Laborales y Recursos Humanos.

En definitiva, las actividades realizadas entre los y las profesoras implicadas en este Proyecto de Innovación Docente han puesto de manifiesto la demanda de formación y la necesidad de adecuar las asignaturas implicadas para la adopción de la perspectiva de género; necesidades verbalizadas por el 
profesorado a través de los seminarios realizados, así como a través de las iniciativas de reelaboración de los recursos docentes por su parte, incorporando recursos de aprendizaje que no hagan tratamiento discriminatorio, la utilización de un uso no sexista del lenguaje, la inclusión de actividades que incorporen la perspectiva de género o la inclusión de dinámicas o prácticas que trasladen al alumnado la relevancia de hacer presente la perspectiva de género a lo largo de todo el proceso docente y no solamente mediante actividad aisladas.

\section{Referencias}

AGÈNCIA PER A LA QUALITAT DEL SISTEMA UNIVERSITARI DE CATALUNYA (2018). Marc general per a la incorporació de la perspectiva de gènere en la docència. $<\mathrm{http} / / /$ www.aqu.cat/universitats/mggenere.html\#.XxahbOcp5PY> [Consulta: 20 de septiembre de 2019]

GUNI (2017). Higher Education in the World 6. Towards a Socially Responsible University: Balancing the Global with the Local. <http://www.guninetwork.org/report/higher-education-world-6> [Consulta: 20 de junio de 2019]

MAGALLÓN, C. (2016). "Ciència des de les vides de les dones, millor ciència? com la investigació amb perspectiva de gènere millora les ciències i les vides" en Mètode Science Studies Journal, No 91 p. 57-63.

PÉREZ SEDEÑO, E. (2018). "Conocimiento y Educación Superior desde la perspectiva de género: sociología, políticas públicas y epistemología" en ArtefaCToS. Revista de estudios de la ciencia y la tecnología, Vol. 7, No. 1 (2018), $2^{\mathrm{a}}$ Época, 121-142.

SCHIEBINGER, L. (2008). Gender Innovations in Science and Engineering. Standford: Standford University Press.

TORRES DÍAZ, M. C. (2018). Dret i criminología: Guies per a una docència universitària amb perspectiva de gènere, Xarxa Vives d'Universitats <https://www.vives.org/book/dret-icriminologia-guia-per-a-docencia-universitaria-amb-perspectiva-genere/> [Consulta: 15 de mayo de 2019]

VERGE MESTRE, T. y CABRUJA UBACH, T. (2017). La perspectiva de gènere en docència i recerca a les universitats de la Xarxa Vives: Situació actual i reptes de futur, Xarxa Vives d'Universitats $<$ https://www.vives.org/book/perspectiva-de-genere-en-docencia-i-recerca-a-les-universitatssituacio-actual-i-reptes-de-futur/> [Consulta: 15 de mayo de 2019]

VERGE MESTRE, T. y A. ALONSO ÁLVAREZ. (2019). "La ceguera al género en el currículum de la ciencia política y su impacto en el alumnado"en Revista Internacional de Sociología, 77(3):e135. 